AMILCAR BARRETA ${ }^{1}$

JoAna Fróes Bragança Bastos ${ }^{1}$

LUIS Otávio SARIAN ${ }^{1}$

Maria Carolina Szymanski de Toledo' ${ }^{1}$

Luis Felipe Sallum ${ }^{1}$

SOPHIE DERChAIN ${ }^{1}$

Artigo Original

Palavras-chave

Laparoscopia/efeitos adversos Neoplasias ovarianas/diagnóstico Laparotomia/efeitos adversos

Fatores de risco

Keywords

Laparoscopy/adverse effects Ovarian neoplasms/diagnosis Laparotomy/adverse effects Risk factors

\title{
Laparoscopia na abordagem inicial de tumores anexiais
}

\section{Laparoscopy for diagnosis and treatment of adnexal masses}

\section{Resumo}

OBJETIVO: Avaliar o uso da laparoscopia como método diagnóstico e terapêutico na abordagem inicial de tumores anexiais em população de risco para malignidade, bem como fatores clínicos associados à falha do método e conversão para laparotomia, e comparar taxas de complicação com pacientes cuja abordagem inicial se deu por laparotomia. MÉTODOS: Estudo prospectivo com 210 mulheres com exames de imagem prévios constando tumor anexial, das quais 133 foram incluídas. Destas, 45 foram submetidas à laparoscopia e 88, à laparotomia. Catorze das 45 cirurgias iniciadas por laparoscopia foram convertidas a laparotomia no intraoperatório. Foi avaliado se idade, índice de massa corporal (IMC), número de cirurgias abdominais prévias, CA-125, índice de risco de malignidade (IRM), diâmetro do tumor, duração da cirurgia e número de complicações cirúrgicas diferiram entre os grupos laparoscopia, laparoscopia com conversão à laparotomia e laparotomia. Foi também avaliado o motivo reportado pelos cirurgiões como falha da laparoscopia e a razão da conversão para laparołomia. RESULTADOS: A taxa de tumores malignos neste estudo foi de 30\%. Houve diferença nos valores de CA-125, IRM e diâmetro do tumor entre os grupos laparoscopia e laparotomia. A duração da cirurgia foi maior no grupo de laparoscopias convertidas à laparotomia, porém as taxas de complicação cirúrgica foram semelhantes entre os grupos e, quando isolados os tumores benignos, as taxas de complicação cirúrgica da laparoscopia se mostraram inferiores à laparotomia. Dentre os fatores em estudo, apenas o tamanho do tumor esteve relacionado à conversão para laparotomia. CONCLUSÃO: Este estudo sugere que a abordagem inicial de pacientes com tumores anexiais de risco para malignidade é segura e não aumenta as taxas de complicação, mesmo em pacientes que necessitem de conversão para laparotomia; entretanto, nos casos de dúvida, é preciso avaliar a necessidade de consultar ginecologistas com experiência em laparoscopia avançada e no tratamento de câncer. $\bigcirc$ tamanho do tumor esteve relacionado à conversão para laparotomia.

\section{Abstract}

PURPOSE: To assess clinical factors, histopathologic diagnoses, operative time and differences in complication rates between women undergoing laparoscopy or laparotomy to diagnose and treat an adnexal mass and their association with laparoscopy failure. METHODS: In this prospective study, 210 women were invited to participate and 133 of them were included. Eighty-eight women underwent laparotomy and 45 underwent laparoscopy. Fourteen of the 45 laparoscopies were converted to laparotomy intraoperatively. We assessed whether age, body mass index (BMI), previous abdominal surgeries, CA-125, Index of Risk of Malignancy (IRM), tumor diameter, histological diagnosis, operative time and surgical complication rates differed between the laparoscopy group and the group converted to laparotomy and whether those factors were associated with conversion of laparoscopy to laparotomy. We also assessed surgical logs to evaluate the reasons, as stated by the surgeons, to convert a laparoscopy to laparotomy. RESULTS: In this research, 30\% of the women had malignant tumors. CA-125, IRM, tumor diameter and operative times were higher for the laparotomy group than the laparoscopy group. Complication rates were similar for both groups and also for the successful laparoscopy and unsuccessful laparoscopy groups. The surgical complication rate in women with benign tumors was lower for the laparoscopy group than for the laparotomy group. The factors associated with conversion

Correspondência

Amilcar Barreto Departamento de Ginecologia e Obstetrícia, Faculdade de Ciênnias Médicas, Universidade Estadual de Campinas

Caixa Postal 61

(EP: $13083-970$

Campinas (SP), Brasi

Recebido

$27 / 01 / 2014$

Aceito com modificacoūes $03 / 02 / 2014$
Departamento de Ginecologia e Obstetrícia da Faculdade de Ciências Médicas da Universidade Estadual de Campinas - UNICAMP Campinas (SP), Brasil.

'Departamento de Ginecologia e Obstetrícia, Faculdade de Ciências Médicas, Universidade Estadual de Campinas - UNICAMP Campinas (SP), Brasil.

Conflito de interesses: não há. 
to laparotomy were tumor diameter and malignancy. During laparoscopy, adhesions a large tumor diameter were the principal causes of conversion. CONCLUSION: This study suggests that laparoscopy for the diagnosis and treatment of adnexal masses is safe and does not increase complication rates even in patients who need conversion to laparotomy. However, when doubt about the safety of the procedure and about the presence of malignancy persists, consultation with an expert gynecology-oncologist with experience in advanced laparoscopy is recommended. A large tumor diameter was associated with the necessity of conversion to laparotomy.

\section{Introdução}

Aproximadamente $10 \%$ das mulheres serão submetidas à cirurgia pela presença de um tumor anexial durante sua vida, com uma incidência de câncer muito baixa $^{1,2}$. A Federação Internacional de Ginecologia e Obstetrícia (FIGO) recomenda a laparotomia se, frente a uma paciente com tumor anexial, há forte suspeita de malignidade, fato que está associado a grande morbidade cirúrgica ${ }^{3}$. Na tentativa de redução da morbidade associada ao tratamento cirúrgico, a laparoscopia tem substituído progressivamente a laparotomia como via cirúrgica preferencial, sendo, atualmente, o padrão-ouro para tratamento de tumores anexiais benignos ${ }^{4,5}$. Entretanto, até esta data, estudos que avaliem a segurança e eficácia da laparoscopia no tratamento de tumores anexiais malignos são limitados em tamanho amostral e metodologia ${ }^{6-10}$.

Uma adequada seleção de pacientes com tumor anexial para abordagem cirúrgica inicial por laparoscopia reduz o risco de um tratamento inadequado e permite que a maioria dos tumores anexiais seja tratada por laparoscopia, reduzindo a morbidade cirúrgica, a necessidade de conversão para laparotomia e o achado inesperado de malignida$\mathrm{de}^{10-14}$. Permanece incerto, entretanto, se fatores clínicos comumente usados na determinação de quais casos devam ser operados por laparoscopia realmente estão associados ao sucesso cirúrgico. Atualmente, é investigado se fatores como idade da paciente, índice de massa corporal (IMC), presença e número de cirurgias intra-abdominais prévias, dosagem do CA-125, índice de risco de malignidade (IRM), tamanho tumoral e presença de aderências podem afetar o resultado da laparoscopia ${ }^{15-20}$.

Assim, não está claramente definido se os fatores clínicos usados pelos ginecologistas para seleção de casos de tumor anexial para abordagem cirúrgica inicial por laparoscopia, baseados em sua experiência pessoal, realmente estão relacionados às taxas de sucesso da laparoscopia.

O melhor entendimento sobre quais os fatores clínicos e intraoperatórios que realmente influenciam a adequada seleção de pacientes com tumor anexial para laparoscopia e, consequentemente, as taxas de sucesso da cirurgia podem ajudar os ginecologistas a tomarem melhores decisões quanto à via de escolha para abordagem cirúrgica inicial nesses casos e também para orientação às pacientes sobre os possíveis resultados a serem esperados.

Desta maneira, este estudo foi desenhado para avaliar quais fatores clínicos e intraoperatórios influenciam diretamente nas taxas de sucesso, complicações e na conversão para laparotomia de cirurgias para tratamento e diagnóstico de tumores anexiais inicialmente realizadas por laparoscopia.

\section{Métodos}

Este foi um estudo prospectivo, realizado no Departamento de Ginecologia e Obstetrícia da Faculdade de Ciências Médicas da Universidade de Campinas (UNICAMP), entre janeiro de 2010 e março de 2011. Duzentas e dez mulheres encaminhadas para o ambulatório de oncologia pélvica do Centro de Atenção Integral à Saúde da Mulher (CAISM) da UNICAMP, devido à presença de tumores anexiais previamente identificados em exame clínico e/ou exame de imagem, foram convidadas a participar. Após a entrevista inicial e explicação sobre os métodos e propósitos do estudo, todas as mulheres que aceitaram participar do estudo assinaram um termo de consentimento informado. $\mathrm{O}$ estudo foi aprovado pelo Comitê de Ética em Pesquisa da UNICAMP (CEP 006/2010).

A avaliação inicial das mulheres compreendeu uma ultrassonografia pélvica transvaginal (UPTV) e dosagem de CA-125. Após a avaliação inicial, 77 mulheres foram excluídas pelas seguintes razões: em 24 a UPTV revelou cistos simples, com probabilidade muito baixa de neoplasia; 22 casos não apresentavam massas anexiais na UPTV; 12 perderam seguimento; 6 morreram antes da cirurgia; 4 tiveram tumores diagnosticados em outros órgãos; 4 foram diagnosticadas por biópsia não cirúrgica; 4 foram submetidas a cirurgia em outra instituição e 1 ficou grávida antes da cirurgia (Figura 1).

Após a avaliação inicial, 133 mulheres foram incluídas e tiveram indicação de cirurgia para diagnosticar e/ou tratar uma massa anexial. A decisão sobre a realização de laparoscopia ou laparotomia como abordagem cirúrgica inicial foi baseada na avaliação pré-operatória realizada pelos cirurgiões. O protocolo do estudo não determinava qual abordagem a ser realizada. As cirurgias foram realizadas por um especialista em oncologia ginecológica assistido por um ou mais médicos residentes ou pós-graduandos.

As laparoscopias foram realizadas sob anestesia geral. $\mathrm{O}$ acesso inicial para laparoscopia foi feito com agulha de Verres na maioria dos casos. Em alguns casos, optou-se pela técnica de acesso aberto. Após o estabelecimento do pneumoperitônio, 1 a 4 portais acessórios de 5 a $10 \mathrm{~mm}$ foram introduzidos na cavidade peritoneal. As laparotomias foram realizadas por 
incisão mediana, sob anestesia geral ou regional. A maioria das peças cirúrgicas foi enviada para biópsia de congelação e todas as amostras foram enviadas para exame anátomo-patológico confirmatório em parafina. Todos os exames histopatológicos foram realizados no Departamento de Anatomia Patológica da UNICAMP, seguindo a Classificação da Organização Mundial da Saúde $(\mathrm{OMS})^{21}$. Para a análise estatística, tumores borderline foram classificados como malignos.

Foi avaliado se idade, IMC (em $\left.\mathrm{kg} / \mathrm{m}^{2}\right)$, número de cirurgias abdominais prévias (abertas ou endoscópicas), dosagem de CA-125, IRM, diâmetro do tumor (medida na peça cirúrgica), diagnóstico histopatológico, duração da cirurgia e incidência de complicações cirúrgicas diferiram entre os grupos laparoscopia e laparotomia. Em seguida, foi avaliada a associação desses fatores com a conversão de uma laparoscopia em laparotomia. Neste estudo, o IRM foi calculado por método previamente validado em mulheres brasileiras ${ }^{22}$, tendo sido multiplicado um escore ultrassonográfico (US) com base nas características do tumor (variando de 0 a 10) pelo valor absoluto do CA-125 (em U/mL) e por um fator de correção a depender de a mulher encontrar-se na pré-menopausa (1) ou pós-menopausa (3), o que resulta na relação IRM $=$ USxCA$125 \times \mathrm{xM}$ (resultados variam de 0 a $\infty$ ).

Os registros cirúrgicos presentes nos prontuários médicos foram também avaliados para serem obtidos os motivos, segundo anotações dos cirurgiões, das conversões de uma laparoscopia em laparotomia e a presença de complicações cirúrgicas. As complicações cirúrgicas foram divididas em dois grupos. Consideramos complicações menores: morbidade febril (duas medições de temperatura $\geq 38^{\circ} \mathrm{C}$ em um intervalo de pelo menos 6 horas, no prazo de 48 horas da cirurgia), infecções de ferida operatória, deiscência superficial das suturas, Síndrome da Resposta Inflamatória Sistêmica leve (SIRS) — definida como elevação transitória e/ou moderada da creatinina sérica, ureia ou enzimas hepáticas, ou desequilíbrio hidroeletrolítico que não precisou de correção intravenosa, e ruptura do cisto. Classificamos como complicações maiores: lesões intestinais, do trato urinário ou outras lesões em órgãos intra-abdominais, evisceração, sangramentos maciços (com necessidade de transfusão), trombose e tromboembolismo e SIRS grave com internação em Unidade de Terapia Intensiva (UTI).

Os dados obtidos foram armazenados em planilhas Microsoft Excel ${ }^{\circledR}$ e analisados com o ambiente "R" para estatística computacional ${ }^{23}$. Valores de $\mathrm{p} \leq 0,05$ foram considerados estatisticamente significantes. Foram usados os testes $\chi^{2}$ e/ou teste exato de Fisher e teste de Kruskal-Wallis para comparar as características clínicas das mulheres nos grupos laparoscopia, laparotomia e laparoscopia com conversão em laparotomia.

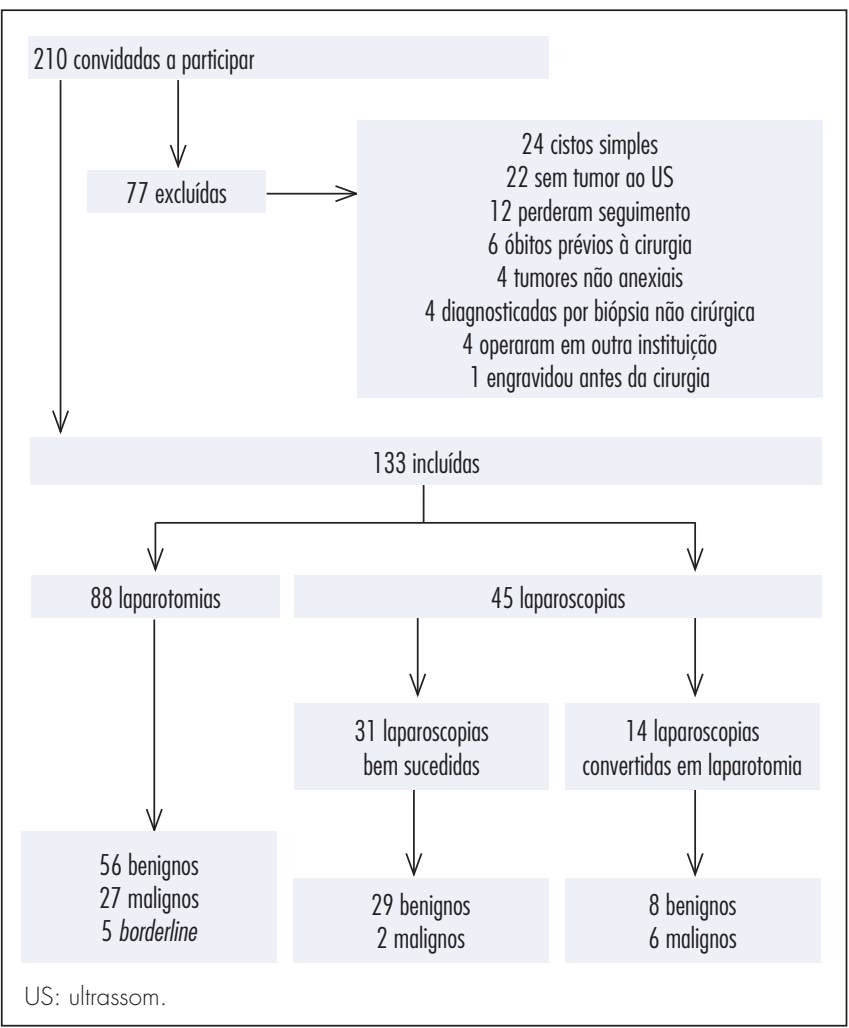

Figura 1. Esquema de seleção das pacientes do estudo.

\section{Resultados}

Nas 133 mulheres incluídas, a abordagem cirúrgica inicial por laparotomia foi escolhida em 88 (66\%) casos e, por laparoscopia, em 45 (34\%). Cinquenta e seis mulheres no grupo laparotomia tinham tumores benignos, 27 tumores malignos e 5 tiveram tumores borderline na histologia final. Das 45 laparoscopias, 31 foram bem sucedidas. Nesses casos, 29 mulheres tinham tumores benignos e 2 tinham tumores malignos. Catorze das 45 laparoscopias foram convertidas em laparotomia. Nestas, 8 mulheres apresentaram tumores benignos e 6 , tumores malignos (Figura 1). A prevalência de tumores malignos em nosso estudo foi de $30 \%$.

As características clínicas das mulheres nos grupos laparoscopia e laparotomia não diferiram com relação à idade (média de 48 versus 51 anos), IMC (média de 27 versus $\left.28 \mathrm{~kg} / \mathrm{m}^{2}\right)$ e no número de cirurgias abdominais anteriores (0 a 1: $71 \%$ das laparoscopias versus $67 \%$ das laparotomias; 2 ou mais: 29 versus $33 \%$, respectivamente). As mulheres do grupo laparoscopia tinham valores mais baixos de CA-125 (média de 33 versus $228 \mathrm{U} / \mathrm{mL}$ ), de IRM (167 versus 2.334 ) e tumores menores (média do maior diâmetro: 6,0 versus $11,5 \mathrm{~cm}$ ). O número de tumores malignos em mulheres que se submeteram à laparoscopia foi significativamente menor do que entre as mulheres submetidas à laparotomia ( $8 / 45$ versus $32 / 88 ; \mathrm{p}=0,04)$. 
A duração da cirurgia foi, na média, menor para o grupo de laparoscopia em comparação com o grupo de laparotomia (126 versus 155 minutos). A frequência de complicações maiores e menores foi estatisticamente semelhante para os grupos de laparoscopia e laparotomia (Tabela 1).

Restringindo nossas análises aos casos de tumores benignos, a idade ( 47 versus 49 anos), o IMC (27 versus $28 \mathrm{~kg} / \mathrm{m}^{2}$ ) e o número de cirurgias abdominais prévias (0 a 1: $78 \%$ no grupo de laparoscopia versus $62 \%$ no grupo laparotomia; 2 ou mais: 22 versus 38\%, respectivamente) não diferiram entre os grupos laparoscopia e laparotomia. Os valores de CA-125 (14 versus $88 \mathrm{U} / \mathrm{mL}$ ) e do IRM (30 versus 373) e a medida do maior diâmetro do tumor $(5,5$ versus $11,0 \mathrm{~cm})$ foram significativamente menores para o grupo de laparoscopia em comparação ao grupo laparotomia. A duração da cirurgia foi menor (108 versus 144 minutos) e as complicações menores foram menos frequentes no grupo laparoscopia em relação ao grupo laparotomia (3 versus $17 \%)(\mathrm{p}=0,04)$. A incidência de complicações maiores (0 versus $7 \%$ ) foi semelhante para ambos os grupos (Tabela 2).

$\mathrm{Na}$ Tabela 3, analisamos a taxa de falha (conversão para laparotomia) da laparoscopia. Quatorze (31\%) das 45 cirurgias laparoscópicas foram convertidas em laparotomia.

Tabela 1. Características clínicas das pacientes operadas por laparoscopia e por laparotomia, conforme intencão de tratar

\begin{tabular}{|c|c|c|c|}
\hline \multirow[b]{2}{*}{ Característica } & \multicolumn{3}{|c|}{ Cirurgia (conforme intenção de frafar) } \\
\hline & $\begin{array}{l}\text { Laparoscopia } \\
(n=45)\end{array}$ & $\begin{array}{l}\text { Laparotomia } \\
(\mathrm{n}=88)\end{array}$ & Valor $p$ \\
\hline \multicolumn{4}{|l|}{ Idade (anos) } \\
\hline Média $\pm \mathrm{DP}$ & $48 \pm 15$ & $51 \pm 15$ & 0,5 \\
\hline \multicolumn{4}{|l|}{ IMC ( $\left(\mathrm{kg} / \mathrm{m}^{2}\right)$} \\
\hline Média \pm DP & $27 \pm 4$ & $28 \pm 5$ & 0,2 \\
\hline \multicolumn{4}{|c|}{ Cirurgias abdominais prévias - n (\%) } \\
\hline 0 a 1 & $32(71)$ & $59(67)$ & 0,6 \\
\hline 2 ou mais & $13(29)$ & $29(33)$ & \\
\hline \multicolumn{4}{|c|}{ CA-125 (U/mL) } \\
\hline Média $\pm D P$ & $33 \pm 71$ & $228 \pm 562$ & $<0,001$ \\
\hline \multicolumn{4}{|l|}{ IRM } \\
\hline Média $\pm D P$ & $167 \pm 755$ & $2.334 \pm 9.831$ & $<0,001$ \\
\hline \multicolumn{4}{|c|}{ Maior diâmetro do fumor $(\mathrm{cm})$} \\
\hline Média $\pm D P$ & $6 \pm 3$ & $11,5 \pm 6,5$ & $<0,001$ \\
\hline \multicolumn{4}{|c|}{ Diagnóstico histológico - n (\%) } \\
\hline Benigno & $37(82)$ & $56(64)$ & 0,04 \\
\hline Borderline & 0 & - & $5(6)$ \\
\hline Maligno & $8(18)$ & $27(30)$ & \\
\hline \multicolumn{4}{|c|}{ Duração da cirurgia (minutos) } \\
\hline Média $\pm D P$ & $126 \pm 8$ & $155 \pm 82$ & 0,03 \\
\hline \multicolumn{4}{|c|}{ Complicações - n(\%) } \\
\hline Menores & $3(6)$ & $12(14)$ & 0,3 \\
\hline Maiores & $4(9)$ & $12(14)$ & 0,6 \\
\hline
\end{tabular}

\#Conforme abordagem cirúrgica inicial.

DP: desvio-padrão; IMC: índice de massa corporal; IRM: índice de risco de malignidade.
Tabela 2. Características clínicas das pacientes com histologia benigna operadas por laparoscopia e por laparotomia, conforme intenção de tratar

\begin{tabular}{|c|c|c|c|}
\hline \multirow[b]{2}{*}{ Característica } & \multicolumn{3}{|c|}{ Cirurgia (conforme intenção de trafar)\# } \\
\hline & $\begin{array}{l}\text { Laparoscopia } \\
\qquad(n=37)\end{array}$ & $\begin{array}{l}\text { Laparofomia } \\
(\mathrm{n}=56)\end{array}$ & Valor p \\
\hline \multicolumn{4}{|l|}{ Idade (anos) } \\
\hline Média $\pm D P$ & $47 \pm 15$ & $49 \pm 14$ & 0,6 \\
\hline \multicolumn{4}{|l|}{ IMC ( $\left(\mathrm{kg} / \mathrm{m}^{2}\right)$} \\
\hline Média $\pm D P$ & $27 \pm 5$ & $28 \pm 5$ & 0,2 \\
\hline \multicolumn{4}{|c|}{ Cirurgias abdominais prévias - n (\%) } \\
\hline 0 a 1 & $29(78)$ & $35(62)$ & 0,1 \\
\hline 2 ou mais & $8(22)$ & $21(38)$ & \\
\hline \multicolumn{4}{|c|}{ CA-125 (U/mL) } \\
\hline Média $\pm D P$ & $14 \pm 14$ & $88 \pm 313$ & $<0,001$ \\
\hline \multicolumn{4}{|l|}{ IRM } \\
\hline Média $\pm D P$ & $30 \pm 61$ & $373 \pm 1.758$ & 0,005 \\
\hline \multicolumn{4}{|c|}{ Maior diâmetro do tumor (cm) } \\
\hline Média $\pm D P$ & $5,5 \pm 2$ & $11 \pm 5,5$ & $<0,001$ \\
\hline \multicolumn{4}{|c|}{ Duração da cirurgia (minutos) } \\
\hline Média $\pm D P$ & $108 \pm 64$ & $144 \pm 70$ & 0,007 \\
\hline \multicolumn{4}{|c|}{ Complicações - n(\%) } \\
\hline Menores & $1(3)$ & $9(17)$ & 0,04 \\
\hline Maiores & 0 & $4(7)$ & 0,1 \\
\hline
\end{tabular}

Tabela 3. Características clínicas de pacientes submetidas à laparoscopias bem sucedidas comparadas com aquelas que necessitaram de conversão

\begin{tabular}{|c|c|c|c|}
\hline Característica & $\begin{array}{l}\text { Laparoscopia } \\
\qquad(n=31)\end{array}$ & $\begin{array}{l}\text { Conversão em } \\
\text { laparotomia } \\
(n=14)\end{array}$ & Valor $p$ \\
\hline \multicolumn{4}{|l|}{ Idade (anos) } \\
\hline Média $\pm D P$ & $47 \pm 15$ & $51 \pm 14$ & 0,5 \\
\hline \multicolumn{4}{|l|}{ IMC (kg/m²) } \\
\hline Média $\pm D P$ & $27 \pm 5$ & $27 \pm 4$ & 0,6 \\
\hline \multicolumn{4}{|c|}{ Cirurgias abdominais prévias - n (\%) } \\
\hline 0 a 1 & $23(74)$ & $9(64)$ & 0,5 \\
\hline 2 ou mais & $8(26)$ & $5(36)$ & \\
\hline \multicolumn{4}{|c|}{ Maior diâmetro do fumor (cm) } \\
\hline Média \pm DP & $5 \pm 2$ & $8 \pm 3,5$ & 0,006 \\
\hline \multicolumn{4}{|c|}{ Diagnóstico histológico - n (\%) } \\
\hline Benigno & $29(94)$ & $8(57)$ & 0,006 \\
\hline Borderline & 0 & 0 & \\
\hline Maligno & $2^{*}(6)$ & $6(43)$ & \\
\hline \multicolumn{4}{|c|}{ Duração da cirurgia (minutos) } \\
\hline Média $\pm D P$ & $105 \pm 58$ & $174 \pm 100$ & 0,03 \\
\hline \multicolumn{4}{|c|}{ Complicações - n (\%) } \\
\hline Menores & $1(3)$ & $3(21)$ & 0,08 \\
\hline Maiores & $1(3)$ & $2(14)$ & 0,2 \\
\hline
\end{tabular}

\#Um caso de metástase de câncer de mama e um caso de tumor irressecável.

DP: desvio-padrão; IMC: índice de massa corporal. 
Idade ( 47 versus 51 anos), IMC (27 kg/m² em ambos os grupos), número de cirurgias abdominais prévias (0 a 1 : $74 \%$ no grupo laparoscopia versus $64 \%$ no grupo laparotomia; 2 ou mais: 26 versus $36 \%$, respectivamente), CA-125 (19 versus $63 \mathrm{U} / \mathrm{mL}$ ) e IRM (193 versus 108) não diferiram entre os grupos. O maior diâmetro do tumor $(5,0$ versus $8,0 \mathrm{~cm})$ e a presença de um tumor maligno (6 versus 43\%) foram os fatores associados com risco aumentado de conversão. A conversão de uma laparoscopia em laparotomia aumentou significativamente a duração da cirurgia (105 versus 174 minutos) $(\mathrm{p}=0,03)$. No entanto, as taxas de complicação cirúrgica permaneceram semelhantes.

A Tabela 4 mostra as causas intraoperatórias, conforme descrito nos prontuários médicos, para conversão de uma laparoscopia em laparotomia. Conversões foram necessárias devido a dificuldades técnicas antes de ser obtida a amostra para análise histopatológica (12 casos) ou para o estadiamento de um tumor maligno diagnosticado na biópsia de congelação (2 casos). Dentre os casos convertidos por dificuldades técnicas observadas antes do diagnóstico histológico na biópsia de congelação, cinco laparoscopias foram convertidas devido à presença de um tumor de grande volume; um desses casos teve também uma lesão de bexiga urinária. Outros cinco casos foram convertidos devido à presença de aderências que impediram a dissecção e o acesso ao tumor, e um desses casos também cursou com perfuração intestinal. Um caso cursou com lesão de bexiga urinária e a cirurgia foi convertida para permitir a reparação adequada. A cirurgia em uma paciente obesa $\left(\mathrm{IMC}=34,4 \mathrm{~kg} / \mathrm{m}^{2}\right)$ teve de ser convertida devido à dificuldade ventilatória ocorrida após a realização do pneumoperitôneo. A biópsia de congelação revelou neoplasia maligna em três dos casos convertidos devido à presença de aderências, que, portanto, precisariam ser convertidos para o estadiamento, e em um caso de tumor de grandes dimensões, diagnosticado como uma metástase de carcinoma de mama.

Tabela 4. Causas de conversão em laparotomia conforme relato na descrição cirúrgica presente nos prontuários médicos

\begin{tabular}{lccc}
\hline \multirow{2}{*}{ Causa } & $\begin{array}{c}\text { Total } \\
\text { convertido }(\mathrm{n})\end{array}$ & \multicolumn{2}{c}{ Diagnóstico histológico (n) } \\
\cline { 3 - 4 } & 5 & Benigno & Maligno \\
\hline Volume do fumor & 2 & 2 & $1^{*}$ \\
Aderências & 2 & 0 & 3 \\
Estadiamento & 1 & 1 & 2 \\
Complicações cirúrgicas & 1 & 1 & 0 \\
Obesidade & 14 & 8 & 0 \\
Total & 1 & 8 & 6 \\
\hline
\end{tabular}

"Caso convertido por "tamanho do tumor", cujo diagnóstico após a biópsia de congelação foi de metástase de câncer de mama.

Conversões devido a "volume do tumor", "aderências", "complicações cirúrgicas" e "obesidade" ocorreram antes da obtenção de amostras para biópsia de congelação. Conversões para "estadiamento" foram realizadas após um resultado de tumor maligno na biópsia de congelação.

\section{Discussão}

Neste estudo, procuramos examinar os fatores que podem influenciar as taxas de sucesso e, consequentemente, de conversão em laparotomia, da abordagem inicial por laparoscopia de tumores anexiais de risco para malignidade. É importante enfatizar que o melhor resultado cirúrgico é obtido quando há precisa indicação da via cirúrgica previamente ao procedimento; assim, um de nossos principais objetivos foi identificar os fatores clínicos pré-operatórios que aumentam o risco de conversão de uma laparoscopia em laparotomia. Outro objetivo foi identificar os fatores intraoperatórios que aumentam esse risco. Com base no conhecimento desses fatores, os cirurgiões ginecológicos poderiam realizar uma melhor avaliação sobre qual via cirúrgica escolher para abordagem inicial de tumores anexiais, bem como melhor orientar suas pacientes sobre a possibilidade de cirurgia laparoscópica nesses casos.

Um dos principais achados de nosso estudo é que muitas das características clínicas das pacientes, dentre elas, idade, IMC, número de cirurgias abdominais prévias e níveis pouco elevados de CA-125, não necessariamente aumentam as taxas de falha da laparoscopia. $\mathrm{O}$ avanço no desenvolvimento das técnicas anestésicas e do instrumental cirúrgico laparoscópico, com a existência principalmente de material mais adequado à cirurgia em pacientes obesas e idosas, parece colaborar com a redução das complicações e da necessidade de conversão nessas pacientes, fato que pode ter levado à diferença entre nosso estudo e relatos anteriores ${ }^{11-15,18,19}$. Entretanto, o tamanho do tumor, aderências pélvicas extensas, lesões iatrogênicas e dificuldades inerentes à curva de aprendizado do procedimento estiveram significativamente associados à conversão em laparotomia em nosso estudo.

Em nosso serviço, não é realizado tratamento de tumores anexiais malignos por laparoscopia; esse fato pôde ser evidenciado na comparação das características clínicas de pacientes que foram submetidas à laparoscopia com pacientes submetidas à laparotomia no período em estudo, pois observamos que os cirurgiões tenderam a evitar operar casos de tumores com CA-125 e/ou IRM muito elevados por laparoscopia. Exceções a essa regra são tumores com forte suspeita de irressecabilidade ou de serem metástases de tumores primários de outros órgãos que podem ser encaminhados para laparoscopia diagnóstica. Em nosso serviço, todos os casos nos quais um tumor anexial maligno é diagnosticado por biópsia de congelação no intraoperatório de uma laparoscopia têm a cirurgia convertida em laparotomia para tratamento/ estadiamento cirúrgico. 
Nosso estudo não definiu critérios para seleção da via de abordagem cirúrgica inicial. Deste modo, procuramos avaliar o comportamento esperado no dia a dia dos cirurgiões ginecológicos. A seleção baseada na experiência dos cirurgiões limitou a indicação de laparoscopia a valores de CA-125 na faixa de $0-300 \mathrm{U} / \mathrm{mL}$, fato que reduziu a incidência de tumores malignos nas laparoscopias para $18 \%$ diante de uma incidência global de $30 \%$ de malignidade neste estudo. É necessário lembrar, entretanto, que o protocolo do estudo excluiu pacientes com cistos anexiais simples à UPTV, os quais possuem risco teórico muito baixo para malignidade; assim, neste estudo, focamos a abordagem cirúrgica de tumores anexiais de risco moderado a alto para malignidade. A frequência de malignidade encontrada faz com que seja recomendada atenção aos princípios de cirurgia oncológica em toda paciente com tumor anexial submetida à cirurgia, seja por laparoscopia ou por laparotomia, visto que a ruptura de um tumor maligno no intraoperatório aumenta o estádio do tumor, com possíveis repercussões no prognóstico da doença ${ }^{24}$.

A taxa de conversão em laparotomia das laparoscopias realizadas neste estudo $(31 \%)$ foi superior à encontrada frequentemente na literatura, situada ao redor de $10 \%{ }^{12,13,25,26}$. Essa diferença está relacionada ao fato de outros estudos reportarem populações que incluem todo tipo de cirurgia laparoscópica, enquanto neste estudo focamos apenas em procedimentos de alta e média complexidade utilizados no tratamento de tumores anexiais, associado ao fato de que $18 \%$ de nossas laparoscopias seriam convertidas em laparotomia de qualquer maneira, pela presença de um tumor maligno. Uma atitude cautelosa pode ter contribuído parcialmente com o aumento da taxa de conversão, pois a manipulação excessiva de um tumor pode levar à ruptura do mesmo, com risco de disseminação da doença nos casos de tumor maligno ${ }^{24}$.
Entretanto, é importante notar que, adiante da necessidade de conversão em laparotomia, os resultados deste estudo indicam que a abordagem inicial de tumores anexiais por laparoscopia, embora aumente o tempo de duração da cirurgia, não aumenta as taxas de complicação intra e pós-operatória. Assim, nossos resultados sugerem que pode haver potencial benefício da abordagem inicial por laparoscopia, mesmo em casos de risco para conversão, pois nos casos em que essa dúvida persista e a cirurgia possa ser completada por laparoscopia, encontramos na literatura forte evidência de benefício para as pacientes ${ }^{4,5}$.

Diferentemente de outros estudos ${ }^{19}$, não encontramos evidência de aumento nas taxas de complicação cirúrgica e de conversão em laparotomia pela presença de cirurgias abdominais prévias; entretanto, é importante notar que mulheres com múltiplas cirurgias abdominais prévias compreendem menos de $1 / 3$ da população de nosso estudo. Outro fator que merece atenção é o fato de, dos cinco casos neste estudo convertidos em laparotomia devido à presença de extensas aderências pélvicas, três resultarem no diagnóstico de tumor maligno.

Nossos resultados sugerem que o fator preponderante ao aumento do risco de conversão de uma cirurgia laparoscópica para diagnóstico e tratamento de um tumor anexial é o tamanho do tumor e que a falha da laparoscopia com necessidade de conversão em laparotomia não aumenta as taxas de complicação cirúrgica. Assim, é possível que esse achado encoraje os ginecologistas a serem mais liberais na indicação da laparoscopia nesses casos. Entretanto, a alta incidência de tumores malignos em nossa população reforça a necessidade de atenção aos princípios de cirurgia oncológica em todo caso de tumor anexial e, caso haja suspeita pré-operatória da presença de um tumor maligno, a avaliação de um ginecologista com experiência em oncologia ginecológica é recomendada.

\section{Referências}

1. Jemal A, Bray F, Center MM, Ferlay J, Ward E, Forman D. Global cancer statistics. CA Cancer J Clin. 2011;61 (2):69-90.

2. Hilger WS, Magrina JF, Magtibay PM. Laparoscopic management of the adnexal mass. Clin Obstet Gynecol. 2006;49(3):535-48.

3. Benedet JL, Pecorelli S, Ngan HYS, Hacker NF, editors. Staging classifications and clinical practice guidelines of gynaecological cancers [Internet]. 3rd ed. 2006 [cited 2012 Mar 3]. Available from: http://www.ginecologia.unipd.it/Assistenza-Documenti/Unita $\% 20$ operative/Ginecologia\%200ncologica/staging_booklet.pdf

4. Medeiros LR, Stein AT, Fachel J, Garry R, Furness S. Laparoscopy versus laparotomy for benign ovarian fumor: a systematic review and meta-analysis. Int J Gynecol Cancer. 2008;18(3):387-99.

5. Demir RH, Marchand GJ. Adnexal masses suspected to be benign treated with laparoscopy. JSLS. 2012;16(1):71-84.
6. Liu CS, Nagarsheth NP, Nezhat FR. Laparoscopy and ovarian cancer: a paradigm change in the management of ovarian cancer? J Minim Invasive Gynecol. 2009;16(3):250-62.

7. Lawrie TA, Medeiros LR, Rosa DD, da Rosa MI, Edelweiss MI, Stein AT, et al. Laparoscopy versus laparotomy for FIGO Stage I ovarian cancer. Cochrane Database Syst Rev. 2013;2:CD005344.

8. Park JY, Kim DY, Suh DS, Kim JH, Kim YM, Kim YT, et al. Comparison of laparoscopy and laparotomy in surgical staging of early-stage ovarian and fallopian tubal cancer. Ann Surg Oncol. 2008; 15(7):2012-9.

9. Ghezzi F, Malzoni M, Vizza E, Cromi A, Perone C, Corrado $G$, et al. Laparoscopic staging of early ovarian cancer: results of a multi-institutional cohort study. Ann Surg Oncol. 2012;19(5):1589-94. 
10. Gremeau AS, Bourdel N, Jardon K, Rabischong B, Mage G, Pouly JL, et al. Surgical management of non-epithelial ovarian malignancies: advantages and limitations of laparoscopy. Eur J Obstet Gynecol Reprod Biol. 2014;172:106-10.

11. Liu JH, Zanotti KM. Management of the adnexal mass. Obstet Gynecol. 2011;117(6):1413-28.

12. Sokol Al, Chuang K, Milad MP. Risk factors for conversion to laparotomy during gynecologic laparoscopy. J Am Assoc Gynecol Laparosc. 2003;10(4):469-73.

13. Chi DS, Abu-Rustum NR, Sonoda Y, Awtrey C, Hummer A, Venkatraman ES, et al. Ten-year experience with laparoscopy on a gynecologic oncology service: analysis of risk factors for complications and conversion to laparotomy. Am J Obstet Gynecol. 2004; 191(4): $1138-45$.

14. Fagotti A, Vizzielli G, De laco P, Surico D, Buda A, Mandato VD, et al. A multicentric trial (Olympia-MITO 13) on the accuracy of laparoscopy to assess peritoneal spread in ovarian cancer. Am J Obstet Gynecol. 2013;209(5):462.e 1-1 1.

15. Ghezzi F, Cromi A, Siesto G, Serati M, Bogani G, Sturla D, et al. Use of laparoscopy in older women undergoing gynecologic procedures: is it time to overcome initial concerns? Menopause. 2010;17(1):96-103.

16. Ball A, Bentley JR, O'Connell C, Kieser KE. Choosing the right patient: planning for laparotomy or laparoscopy in the patient with endometrial cancer. J Obstet Gynaecol Can. 201 1;33(5):468-74.

17. Enakpene CA, Omigbodun AO, Goecke TW, Odukogbe AT, Beckmann MW. Preoperative evaluation and triage of women with suspicious adnexal masses using risk of malignancy index. J Obstet Gynaecol Res. 2009;35(1):131-8.

18. Camanni M, Bonino L, Delpiano EM, Migliaretti G, Berchialla P, Deltetto F. Laparoscopy and body mass index: feasibility and outcome in obese patients treated for gynecologic diseases. J Minim Invasive Gynecol. 2010;17(5):576-82.
19. Kumakiri J, Kikuchi I, Kitade M, Kuroda K, Matsuoka S, Tokita S, et al. Incidence of complications during gynecologic laparoscopic surgery in patients after previous laparotomy. J Minim Invasive Gynecol. 2010;17(4):480-6.

20. Shiota $M$, Kotani $Y$, Umemoto $M$, Tobiume $T$, Hoshiai $H$. Study of the correlation between tumor size and cyst rupture in laparotomy and laparoscopy for benign ovarian tumor: is $10 \mathrm{~cm}$ the limit for laparoscopy? J Obstet Gynaecol Res. 2012;38(3):531-4.

21. Scully RE, Bonfiglio TA, Kurman RJ, Silverberg SG, Wilkins EJ. Histological typing of female genital tract tumours. 2nd ed. Berlin: Springer-Verlag; 1994. (World Health Organization. International Histological Classification of Tumours).

22. Torres JC, Derchain SF, Faundes A, Gontijo RC, Martinez EZ, Andrade LA. Risk-of-malignancy index in preoperative evaluation of clinically restricted ovarian cancer. Sao Paulo Med J. 2002; 120(3):72-6.

23. R Development Core Team. R: a language and environment for statistical computing: reference index. Version 2.11.1 [Internet]. 2010 [cited 2011 Nov 17]. Available from: http://www.lsw. uni-heidelberg.de/users/christlieb/teaching/UKStaSS10/Rrefman.pdf

24. Vergote I, De Brabanter J, Fyles A, Bertelsen K, Einhorn N, Sevelda $P$, et al. Prognostic importance of degree of differentiation and cyst rupture in stage I invasive epithelial ovarian carcinoma. Lancet. 2001;357(9251):176-82.

25. Huchon C, Bats AS, Bensaïd C, Junger M, Nos C, Chatellier $G$, et al. Prise en charge des masses annexielles organiques: résultats d'une enquête de pratique. Gynecol Obstet Fertil. 2008;36(11):1084-90.

26. Chapron C, Pierre F, Querleu D, Dubuisson JB. [Complications of laparoscopy in gynecology]. Gynecol Obstet Fertil. $2001 ; 29(9): 605-12$. 